\title{
On the striking similarity between the moments of breakthrough curves for a heterogeneous medium and a homogeneous medium with a matrix diffusion term
}

\author{
X. Sánchez-Vila*, J. Carrera \\ Department of Geotechnical Engineering and Geosciences, Universitat Politècnica de Catalunya, Gran Capita, \\ Jordi Girona 1-3, Barcelona 08034, Spain
}

Received 8 April 2003; revised 28 November 2003; accepted 13 December 2003

\begin{abstract}
A usual method to obtain aquifer parameters is to analyze the moments of the breakthrough curves (BTCs) in tracer tests. The parameters to be estimated in this analysis would depend on the conceptual model adopted. Intuitively, if different processes were considered, the shape of the BTCs should be quite different, and one would tend to think that the time and space evolution of the temporal moments should also be quite different. Contrarily, in this paper, we show that two very different conceptual models of solute transport lead to virtually identical moments of the BTC. The two models selected for this study are the classical advection-dispersion equation with a Fickian macrodispersive term and a homogeneous medium advection model with mass-transfer between mobile and immobile matrix phases, for three different models of matrix shape. In both models, the first three moments are linear with travel distance, while the fourth moment is a second order polynomial. This agreement allows us to choose parameters yielding the same moments in the two models. As we consider two fitting parameters, we select them to match the second and third moment. Match in the first moment is obtained from physical arguments. It turns out that the resulting leading term of the fourth moment is identical for both models. As a direct consequence of this work, it follows that for large travel distances it would not be possible to discriminate between conceptual models using data from a single BTC. (C) 2004 Elsevier B.V. All rights reserved.
\end{abstract}

Keywords: Breakthrough curve; Homogenous medium; Fickian macrodispersive term

\section{Introduction}

Solute transport in heterogeneous media has been the subject of a large amount of research in recent years, both theoretically and in real site applications. Theoretical analysis is generally carried out in

\footnotetext{
* Corresponding author. Tel.: +34-934-011-698; fax: +34-934017-251.

E-mail address: xavier.sanchez-vila@upc.es (X. Sánchez-Vila).
}

a geostatistical frame, where the variables that appear in the transport equation are treated as Spatial Random Functions (Matheron, 1971). This has led to much work oriented to study the behavior of hydraulic heads and/or concentrations and to find the values of effective and equivalent parameters for groundwater flow and solute transport. The working methodologies and the main results are summarized in the books by Dagan (1989), Gelhar (1993) and Zhang (2002) or Rubin (2003). 
One of the most prominent effects of heterogeneity is dispersion. Spatial variability of water velocity causes the solutes to disperse. In fact, one of the most significant findings of stochastic hydrogeology has been the derivation of the equation controlling transport at long distances from the equation controlling transport at the local scale and the assumption of heterogeneous velocity. The usual representation is the advection-dispersion equation (ADE) with a Fickian macrodispersion term

$$
\varphi \frac{\partial\langle C\rangle}{\partial t}+\langle\mathbf{q}\rangle \nabla\langle C\rangle-\nabla(\mathbf{A}\langle\mathbf{q}\rangle \nabla\langle C\rangle)=0,
$$

where $\langle C\rangle$ is the expected value of the concentration of any given solute, $\varphi$ is total porosity, $\langle\mathbf{q}\rangle$ is the

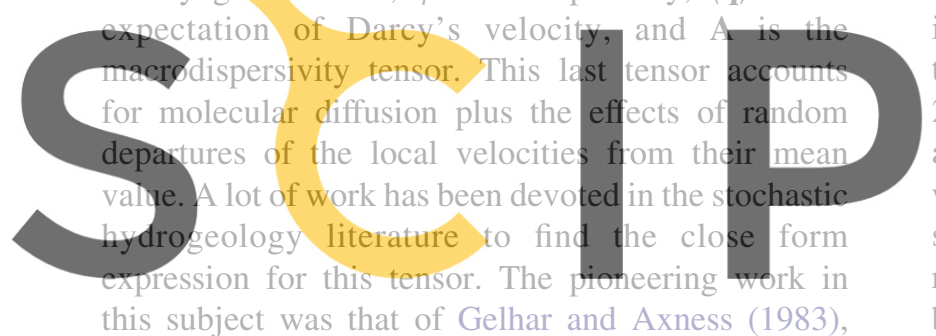

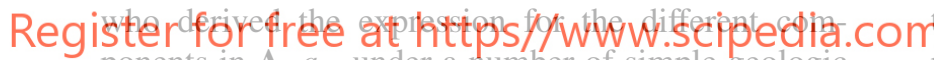

ponents in $\mathbf{A}, a_{i j}$, under a number of simple geologic structures and flow conditions.

This Fickian representation of dispersion is not valid at intermediate distances. In fact, a vast amount of research has been devoted to explaining and representing the well-known scale-dependence of dispersivity. Dagan (1984), under the hypothesis of $\langle C\rangle$ Gaussian, derives a solution where the $a_{i j}$ terms become functions of travel time $(\tau)$ from the source. In particular, the longitudinal dispersivity, $a_{11}$, is found to grow linearly for small and reaches an asymptote for large travel distances (Dagan, 1984). Similar results are obtained by Neuman et al. (1987) using a different approach. Dagan (1987, 1988); Neuman and Zhang (1990) derive the time dependent macrodispersivity tensor from particle displacement moments. Jaekel and Vereecken (1997) applied the renormalization group method to obtain also a scale dependent tensor, still local in space. Their findings were later validated numerically by Schwarze et al. (2001).

Due to the time dependence, different attempts have been made to find asymptotic values for macrodispersion in fields consisting of more than one scale (Zhan and Wheatcraft, 1996; McLaughlin and Ruan, 2001; Lunati et al., 2002). Dagan and Neuman (1991) and a number of authors, thereafter (Cushman and Ginn, 1993; Kavvas and Karakas, 1996; Dentz et al., 2000) derive a different aspect for the equation, where the dispersive term is nonlocal in either space or time (and thus the macrodispersive term is non-Fickian). Several results are also available for transport in non-uniform flow incorporating conditional moments (Neuman, 1993; Butera and Tanda, 1999; Guadagnini and Neuman, 2001).

Unfortunately, the above non-local approaches are difficult to apply in real problems. Therefore, dispersion is still represented by means of a Fickian (macrodispersive) term in most cases. Problems caused by this choice are reviewed by Carrera (1993). Among them, we wish to stress here the inaccurate reproduction of both plumes and break-
through curves (BTCs) (see also Kennedy and Lennox,
2001). Contrary to what Fickian disper ion predicts,
actual plumes often display a highly asymmetric shape,
with the maximum displaced towards the front. By the
same token, Fickian models fitted to early time data
rarely reproduce the lon tails exhibited by late time breakthrough data. As an example, in Fig. 1, we show

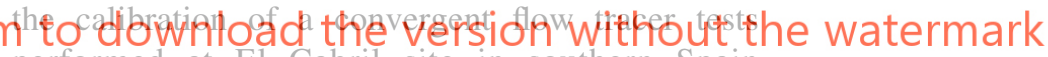
performed at El Cabril site in southern Spain (UPC-UPM, 1991) obtained by fitting early time data with the standard ADE. From the figure, late time data is poorly predicted and the total mass calibrated is lower than the actual input mass.

The two features commented (asymmetric spatial distributions and long tails) can be simulated by means of a term representing the exchange of solute between mobile and immobile regions. This type of formulation is often used to represent matrix diffusion in fractured media (Neretnieks, 1980; Barker and Foster, 1981); sorption (coupled or uncoupled to diffusion) into slowly moving portions of soils (Sposito et al., 1986; Shapiro, 2001; Wörman et al., 2003); non-instantaneous (kinetic) reversible mass transfer (Cunningham et al., 1997; Haggerty and Gorelick, 1998; McKenna et al., 2001); the effect of connectivity of highly conductive features (Zinn and Harvey, 2003); low permeability inclusions (Guswa and Freyberg, 2000); and other phenomena (Zimmerman et al., 2002; Carrera et al., 1998). We will call this term 'matrix diffusion' for shortness, because it can be viewed as representing a diffusive exchange between a flowing portion 

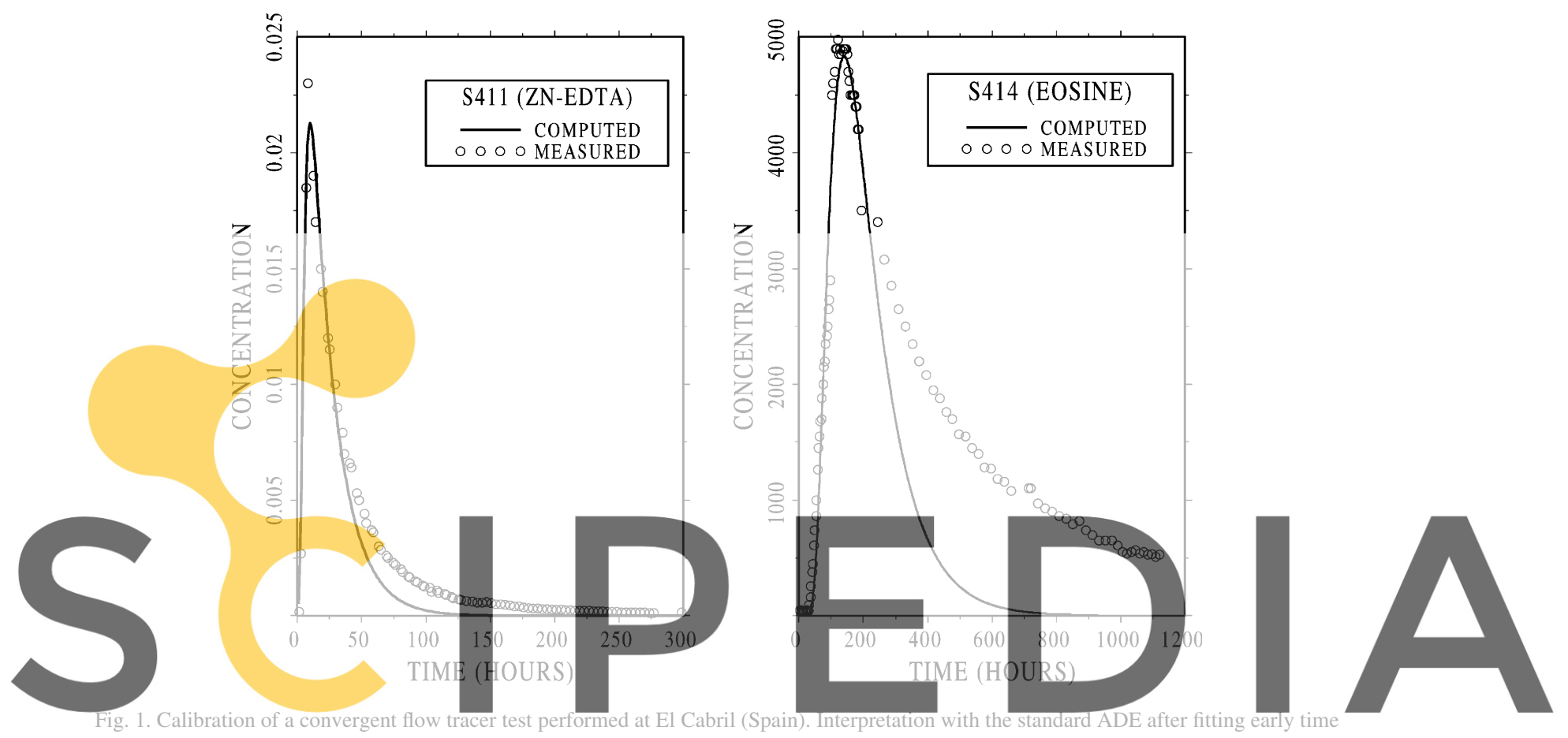

data (adapted from UPC-UPM, 1991). Best fit is obtained with a reduced input mass.

\section{Register for free at https//www.scipedia.co} into the non-flowing portion is delayed, thus leading to asymmetric plumes, reduction in peak concentrations and long-tailed BTCs. In Fig. 2, we see the same BTC than in Fig. 1 now interpreted allowing linear exchange with an immobile water phase (UPC-UPM, 1991). In this case, the peak is again well characterized, but now tailing is well reproduced.

The results shown in Figs. 1 and 2 suggest that a matrix diffusion term could be used for representing dispersion more accurately than a Fickian term, thus providing a better reproduction of transport through heterogeneous media. In fact, Carrera et al. (1998) showed that the matrix diffusion term can be substituted by means of a convolution product resembling analogous terms used by Dagan and Neuman (1991) or by Berkowitz and Scher (1995). Also, Guswa and Freyberg (2002) show that a mass transfer term performed much better than a dispersive term in a bimodal aquifer where low permeability inclusions are found in a high conductive matrix.

The original motivation of our work was to seek a matrix diffusion representation of dispersion that is consistent with stochastic formulations of transport in to download the version without the watermark fitting macrodispersion with a matrix diffusion term, we could reproduce the time evolution of the dispersion coefficient that has been derived by stochastic methods. As it turns out, such a representation is not possible. We will see that the evolution of the BTC moments is not adequate. Still, this representation can be viewed as a convenient alternative to Fickian macrodispersion, that is, valid after ergodic conditions have been attained. One of the critical points is that the parameters included in the mass transfer term should have a physical meaning, and, therefore, should be related to actual parameters characterizing the heterogeneity of the medium.

The approach to reach such objective is as follows. First, we derive the moments of the expected BTC corresponding to a heterogeneous medium assuming that the ADE with a Fickian macrodispersive term holds. Second, we find the travel time moments for a homogeneous medium in which a matrix diffusion term was considered. The last point is to show how a proper identification of parameters leads to identical temporal moments up to fourth order in the case of large travel distances. Finally, we discuss the physical 

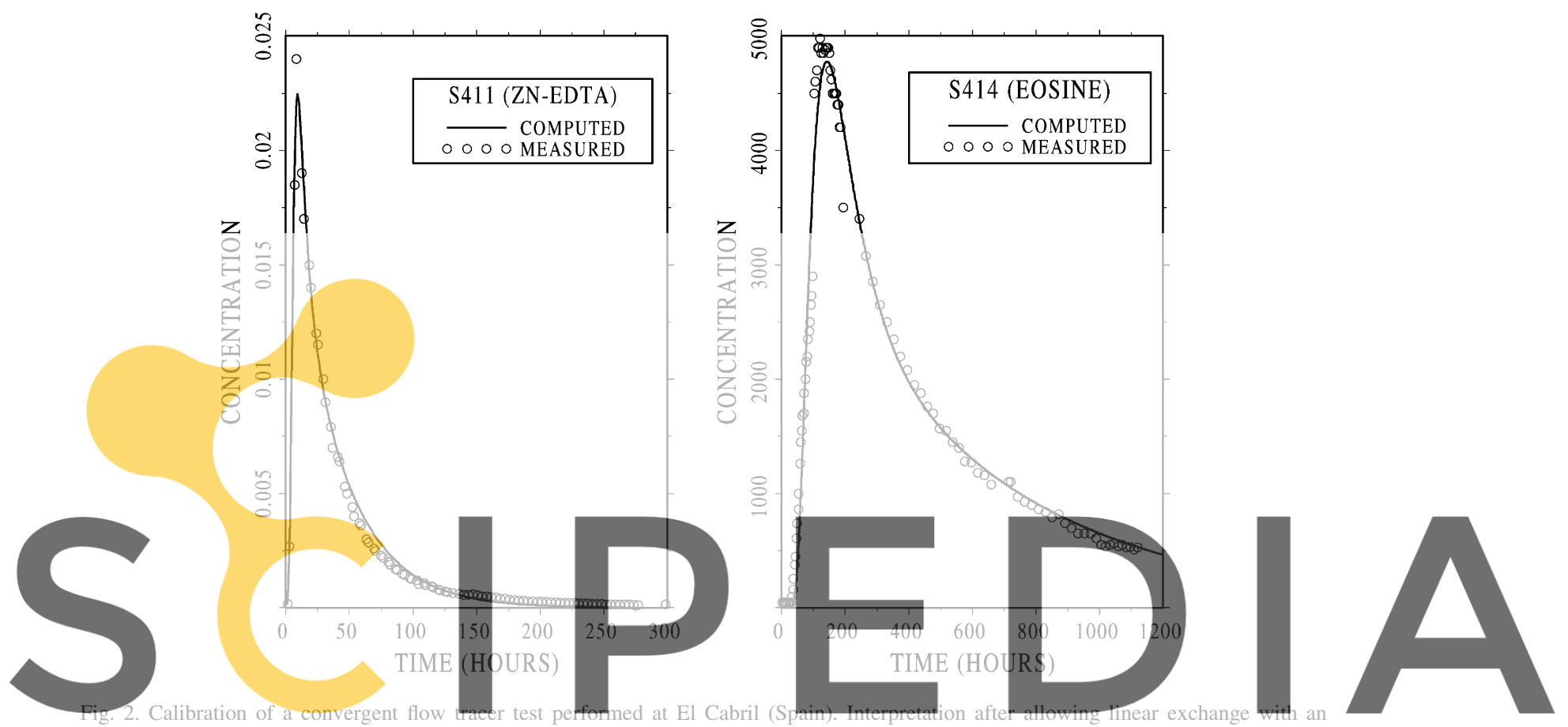

immobile water phase (adapted from UPC-UPM, 1991)

\section{Register for free at https//www.scipedia.col meaning of the identified parameters as well as some implications and extensions of our work.}

\section{Temporal moments for the ADE with a Fickian macrodispersive term}

Under mean uniform flow in the $x$-direction (without any loss of generality), and considering that for steady-state flow and long travel distances the transverse macrodispersivity is negligible with respect to the longitudinal one (Dagan, 1988), we can rewrite Eq. (1) as

$\varphi \frac{\partial\langle C\rangle}{\partial t}+\langle q\rangle \frac{\partial\langle C\rangle}{\partial x}-a_{11}\langle q\rangle \frac{\partial^{2}\langle C\rangle}{\partial x^{2}}=0$,

where $a_{11}$ is (longitudinal) dispersivity and can be written in terms of the parameters that characterize the hydraulic conductivity Spatial Random Function. As an example, for isotropic media $a_{11}=\sigma_{Y}^{2} I$ (Dagan, 1989), where $\sigma_{Y}^{2}$ is the variance of log-conductivity and $I$ is the integral distance in the $x$-direction. We disregard local dispersion, because it is usually very small compared to the macrodispersive term. It m to download the version without the watermark must be pointed that the two simplifications (namely, neglecting transverse dispersion and local dispersion) have been adopted without loss of generaility. That is, they do not affect later results, as we will be interested in travel time towards a plane located perpendicular to the mean flow direction (Cvetkovic et al., 1992), which can be reduced to a single point along the flow direction under these two simplifications. Actually, Berglund and Fiori (1997) showed that pore-scale (local) dispersion affects transport primarily through transverse mixing.

We consider the case of an instantaneous point injection in an initially clean aquifer, which can be taken as a kernel for any other kind of injection in space and time. The goal is to get an expression for the temporal moments of the BTC at a point located at distance $x$. To this end, it is convenient to set the problem in the Laplace space

$\varphi s \bar{C}+\langle q\rangle \frac{\partial \bar{C}}{\partial x}-a_{11}\langle q\rangle \frac{\partial^{2} \bar{C}}{\partial x^{2}}=0$

where $s$ is the Laplace variable and $\bar{C}$ is the Laplace transform of $\langle C\rangle$. The analytical solution for this 
problem becomes:

$\bar{C}(x, s)=\exp \left\{\frac{x}{2 a_{11}}\left(1-\sqrt{1+\frac{4 \varphi a_{11}}{\langle q\rangle} s}\right)\right\}$.

Inversion of Eq. (4), would yield the expected BTC. However, we are only interested in its moments, which are easier to find from the solution of $\mathrm{C}$ in Laplace space. The non-centered temporal moments, defined as

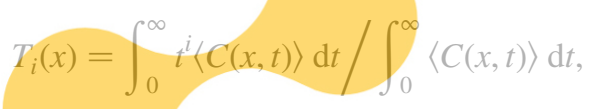

can be obtained by taking the limits of sequential derivatives of Eq. (4) with respect to $s$ (Aris, 1958):

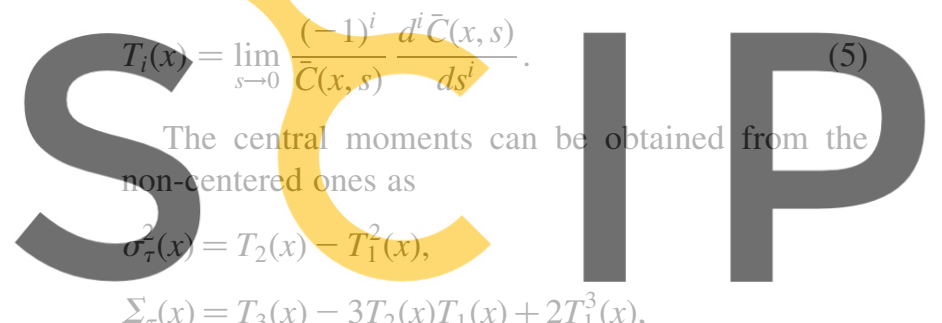

$\Sigma_{\tau}(x)=T_{3}(x)-3 T_{2}(x) T_{1}(x)+2 T_{1}^{3}(x)$,

with $\langle U\rangle=\langle q\rangle / \varphi$ meaning the expected value of the advective velocity. Sequential differentiation of Eq. (7) and substitution in Eqs. (5) and (6) leads to the temporal moments:

$$
\begin{aligned}
T_{1}(x) & =\frac{x}{\langle U\rangle}, \\
\sigma_{\tau}^{2}(x) & =2 \frac{a_{11}}{\langle U\rangle^{2}} x,
\end{aligned}
$$

$\Sigma_{\tau}(x)=12 \frac{a_{11}^{2}}{\langle U\rangle^{3}} x$,

$M_{4, \tau}(x)=12 \frac{a_{11}^{2}}{\langle U\rangle^{4}} x^{2}+120 \frac{a_{11}^{3}}{\langle U\rangle^{4}} x$.

As a result, the first three moments are linear with travel distance, while the fourth order moment is a second order polynomial. Notice that for large distances the leading term in the fourth order moment (8d) is the one in $x^{2}$, but in general there will also be a contribution in $x$. We will come back to this point later.

\section{Homogeneous media with a matrix}
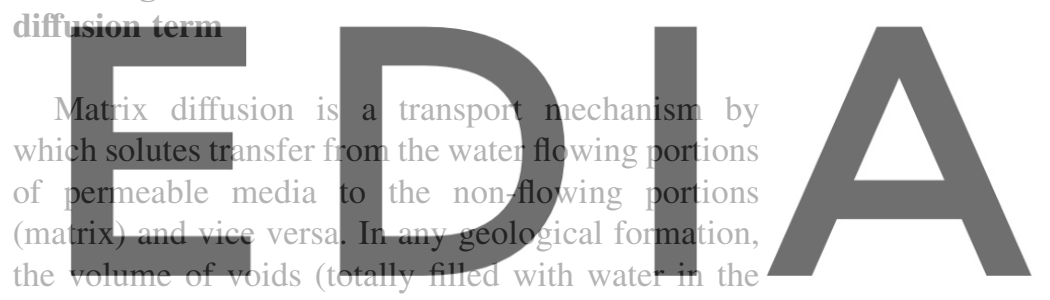

case of saturated flow) can be separated for modeling voids that can be reached by flowing water (mobile zone), while the second represents those that cannot (immobile zone). The solute can only enter this second zone by molecular diffusion. The term matrix diffusion refers to this exchange between mobile and immobile zones (Neretnieks, 1980). While most of the work in matrix diffusion is concentrated on low permeability fractured media (Rasmuson and Neretnieks, 1981; Rasmuson, 1984; Shapiro, 2001; Wörman et al., 2003), this process can also be encountered in granular materials (Wood et al., 1990; Cunningham et al., 1997) or clays (Carrera et al., 1990).

The effects of matrix diffusion are diverse. On one hand, a large volume of voids becomes accessible to the solute by diffusion. This causes an apparent retardation with respect to solutes that do not enter the matrix (Maloszewski and Zuber, 1985; Goltz and Roberts, 1987). An interesting example is that of Zuber et al. (2001), who presented a case in Poland where matrix diffusion causes a huge retardation (on the order of 50) that is assumed to be the cause of detected pollution still not affecting a phreatic aquifer. On the other hand, diffused solutes may take a long 
time to come out of the matrix. This causes tailing in BTCs.

The objective of this section is to find the moments of BTC when we incorporate a term that accounts for diffusion into the matrix instead of the Fickian dispersion term of Eq. (2). Therefore, the processes considered are advection along $x$ in the mobile zone, and mass transfer by diffusion between the mobile and the immobile zones. No retardation is considered in either zone, although it would be straight forwards to consider it in our derivation. Local dispersion is also neglected in order to make it comparable to the ADE case, but again it would be straightforward to incorporate it. The transport equation finally becomes (Carrera et al., 1998)

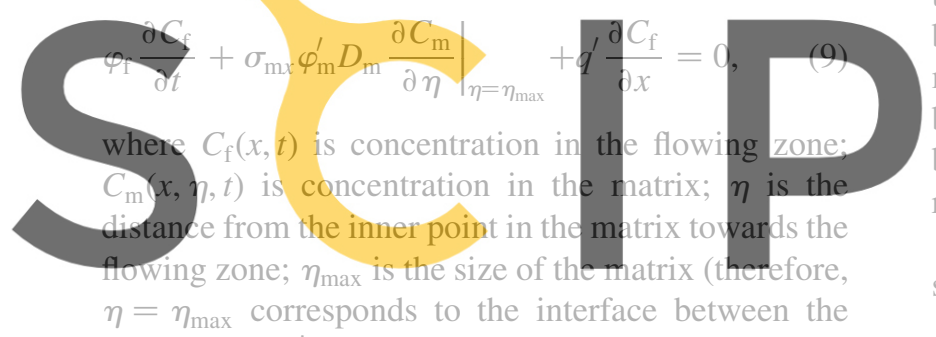

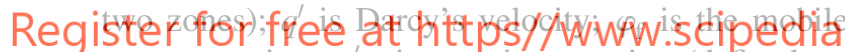

zone porosity; $\varphi_{\mathrm{m}}^{\prime}$ is matrix porosity (defined as volume of voids per unit volume of matrix); $D_{m}$ is the molecular diffusion coefficient; $\sigma_{\mathrm{mx}}$ is the specific surface of the matrix (matrix surface area per unit volume of aquifer) evaluated at the interface. Different variations of Eq. (9) have been used by most authors (Rasmuson and Neretnieks, 1980, 1981; Grisak and Pickens, 1981; Barker, 1982, 1985; Moench, 1984; Sudicky and Frind, 1992; Maloszewski and Zuber, 1993; Novakowski and Lapceviec, 1994; Haggerty and Gorelick, 1995; Kennedy and Lennox, 1995; Wörman et al., 2003, among others).

Concentration in the matrix, $C_{\mathrm{m}}$, is given by the diffusion equation (assuming no retardation)

$\sigma_{\mathrm{m}}(\eta) \frac{\partial C_{\mathrm{m}}}{\partial t}=\frac{\partial}{\partial \eta}\left(D_{\mathrm{m}} \sigma_{\mathrm{m}}(\eta) \frac{\partial C_{\mathrm{m}}}{\partial \eta}\right)$,

where $\sigma_{\mathrm{m}}(\eta)$ is the specific diffusion surface at depth $\eta$, with $\sigma_{\mathrm{m}}\left(\eta_{\max }\right)=\sigma_{\mathrm{mx}}$. There is a direct relationship between the diffusion surface and the model selected for the geometry of the matrix. The three more typical geometries used for the matrix are that of slabs, cylinders, and spheres. In these three cases, $\sigma_{\mathrm{m}}(\eta)$ is proportional to $\eta^{\mathrm{n}}$, with $n=0,1$, and 2 , respectively.
Eq. (10) is solved subject to

$C_{\mathrm{m}}(x, r, t=0)=0$,

$C_{\mathrm{m}}(x, r=1, t)=C_{\mathrm{f}}(x, t) \quad$ at $\Gamma_{\mathrm{m}}$,

$\frac{\partial C_{\mathrm{m}}}{\partial r}(x, r=0, t)=0$

(11c)

where we have introduced a new notation $r=\eta / \eta_{\max }$; Eq. (11a) is the initial condition, which corresponds to initially clean matrix; Eq. (11b) expresses continuity of concentrations at $\Gamma_{\mathrm{m}}$ (or $r=1$ ), which is the interface between the flowing and matrix zones (continuity of mass flux was implicitly imposed in the derivation of Eq. (9)). Finally, Eq. (11c) is the boundary condition at the innermost portion of the
matrix, which depends on the geometry of matrix
blocks. Thus, $r=1$ contesponds to the half distance
between fractures for a slab model, and is equal to the
radius in spheres or cylinders.
Eq. (10) can be solved in Laplace space. After
some minor manipulation Eq. (10) can be rewritten as

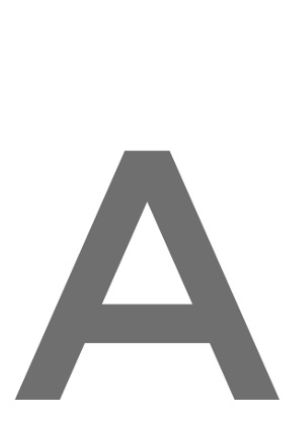

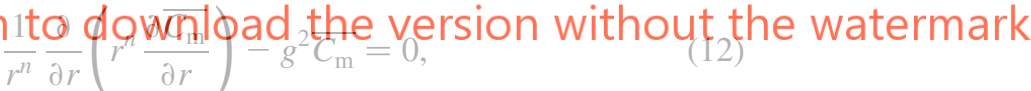
where $g=\sqrt{s / D_{\mathrm{m}}^{\prime}}, s$ being Laplace variable and $D_{\mathrm{m}}^{\prime}=$ $D_{\mathrm{m}} / \eta_{\max }^{2} \cdot \overline{C_{\mathrm{m}}}(x, r, s)$ is the Laplace transform of the concentration in the matrix. The general solution to Eq. (12) subject to BC (Eqs. (11a) and (11c)) and boundness of the solution is given by Gradshteyn and Ryzhik (1980), section 8.49, and taking into account the relationships between Bessel Functions, $J_{\nu}(z)$, and Modified Bessel Functions, $\left.I_{\nu}(z)\right)$

$\overline{C_{\mathrm{m}}}(x, r, s)=r^{\nu} \frac{I_{-\nu}(g r)}{I_{-\nu}(g)} F(x, s)$,

with $\nu=(1-n) / 2$, and $I_{-\nu}(z)$ stands for Modified Bessel function of order $-\nu$. Applying BC (Eq. (11b)), it follows immediately that $F(x, s)=\overline{C_{\mathrm{f}}}(x, s)$, where $\overline{C_{\mathrm{f}}}(x, s)$ is the Laplace transform of the concentration in the mobile phase.

The next step is to go back to the transport Eq. (9) and get a similar expression for the Laplace transform of the concentration of the mobile phase, $\overline{C_{\mathrm{f}}}(x, s)$. Assuming a pulse injection at point $x=0$ (i.e. $C_{\mathrm{f}}(x=0, t)=\delta(t)$, and after some manipulation 
the solution is

$\overline{C_{\mathrm{f}}}(x, s)=\exp \left(-\frac{s}{U^{\prime}} x-(n+1) \frac{\varphi_{\mathrm{m}} D_{\mathrm{m}}^{\prime}}{\varphi_{\mathrm{f}}} \frac{x}{U^{\prime}} g \frac{I_{1-\nu}(g)}{I_{-\nu}(g)}\right)$,

with $U^{\prime}=q^{\prime} / \varphi_{\mathrm{f}}$ and $\varphi_{\mathrm{m}}=\sigma_{\mathrm{mx}} \varphi_{\mathrm{m}}^{\prime} \eta_{\max } /(n+1)$ is the porosity of the matrix (defined as volume of voids per unit volume of aquifer; recall that $\varphi_{\mathrm{m}}^{\prime}$ was expressed per unit volume of matrix).

From here, we can proceed to find the different moments of the BTC. Again a relatively simple way to obtain the different $T_{i}(x)$ terms is to perform a McLaurin expansion around $s=0$ of the exponent in Eq. (14), leading to:

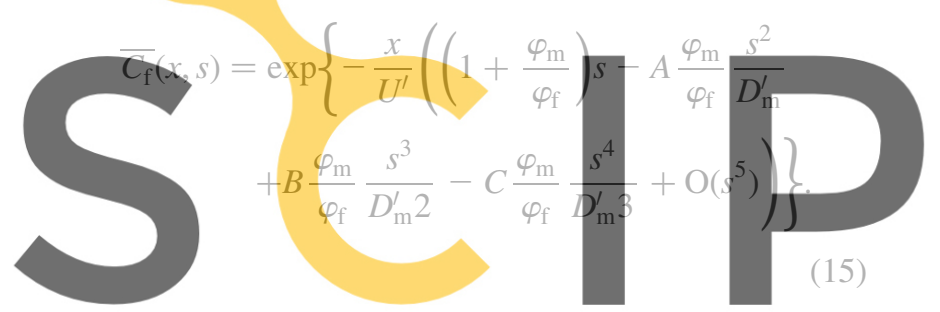

The values for $A, B$ and $C$ depend on

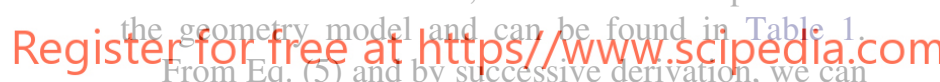
obtain again the temporal moments. The final expressions are:

$T_{1}(x)=\frac{x\left(\varphi_{\mathrm{m}}+\varphi_{\mathrm{f}}\right)}{q^{\prime}}$,

$\sigma_{\tau}^{2}(x)=E \frac{1}{D_{\mathrm{m}}^{\prime}} \frac{\varphi_{\mathrm{m}} x}{q^{\prime}}$

$\Sigma_{\tau}(x)=F \frac{1}{D_{\mathrm{m}^{2}}^{\prime}} \frac{\varphi_{\mathrm{m}} x}{q^{\prime}}$

$M_{4, \tau}(x)=G \frac{1}{D_{\mathrm{m}^{2}}^{\prime}}\left(\frac{\varphi_{\mathrm{m}}}{\varphi_{\mathrm{f}}}\right)^{2}\left(\frac{x}{U^{\prime}}\right)^{2}+H \frac{1}{D_{\mathrm{m}^{3}}^{\prime}} \frac{\varphi_{\mathrm{m}}}{q^{\prime}} x$.

Table 1

Values for $A, B$, and $C$ in Eq. (15) for different matrix shapes

\begin{tabular}{llll}
\hline Matrix shape & $A$ & $B$ & $C$ \\
\hline Slabs & $1 / 3$ & $2 / 15$ & $17 / 315$ \\
Cylinders & $1 / 8$ & $1 / 48$ & $11 / 3072$ \\
Spheres & $1 / 15$ & $2 / 315$ & $1 / 1575$ \\
\hline
\end{tabular}

Table 2

Values for $E, F, G$, and $H$ in Eqs. (16a)-(16d) for different matrix shapes

\begin{tabular}{lllll}
\hline Matrix shape & $E$ & $F$ & $G$ & $H$ \\
\hline Slabs & $2 / 3$ & $4 / 5$ & $4 / 3$ & $136 / 105$ \\
Cylinders & $1 / 4$ & $1 / 8$ & $3 / 16$ & $11 / 128$ \\
Spheres & $2 / 15$ & $4 / 105$ & $4 / 75$ & $8 / 525$ \\
\hline
\end{tabular}

The values for $E, F, G$ and $H$ depend again on the matrix geometry model and are displayed in Table 2 for the three most common ones. The compact expressions presented here would allow obtaining the temporal moments for other geometries in which $\mathrm{n}$
would be a fractional valuc.
Fron Eqs. (16a)-(16c), we see that the mean
arrival time is identical to that of a single porosity
media with the total porosity $\left(\varphi_{\mathrm{m}}+\varphi_{\mathrm{f}}\right)$ of the double
pordsity media. This is independent of the value of the
matix diffusion coefficient, and or matrix block shape and size. This result was also obtained by Goltz and

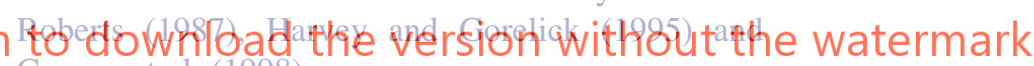
Carrera et al. (1998).

Another interesting result is that the second and third order moments are linear with travel distance, while the fourth order moment is a second order polynomial with respect to $x$. Note that the same behavior was found for the ADE with a Fickian macrodispersive term (Eqs. (8a)-(8d)). This implies that the behavior of the double porosity transport equation is qualitatively similar to that of single porosity with a Fickian dispersion term, at least up to fourth order moments, despite the dissimilarities discussed in the introduction. While this is nice, it disproves the conjecture, mentioned in the introduction, that a matrix diffusion term might display scale dependent dispersion. This would have required the second order moment (Eq. (16b)) to incorporate a non-linear term with a behavior of order $x^{2}$ for short distances (in order to be comparable with the expression presented for example in Cvetkovic et al. (1996)). Still, the equivalence to Eq. (2) suggests that one may choose matrix diffusion parameters to yield the same moments as Fickian transport. This is discussed in Section 4. 


\section{Identification of terms}

\subsection{Identification of the travel time statistical moments}

The objective of this section is to find a set of parameters that makes the first temporal moments identical in both formulations. In the process of fitting parameters, it is necessary to keep invariant some fundamental values. Specifically, we impose that water flux (Darcy velocity) and total porosity should be kept invariant. Therefore, porosity $(\varphi)$ used in Eq. (2) should be equal to total porosity of the double porosity formulation $\left(\varphi_{\mathrm{m}}+\varphi_{\mathrm{f}}\right)$, and both equal to the value measured by field or lab methods. Therefore:
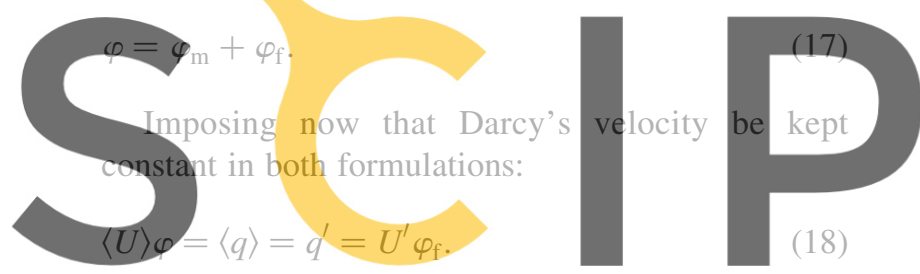

Substituting Eqs. (17) and (18) in the expressions

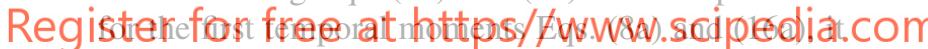

results immediately that they are equal. That is, ensuring that water flux and total porosity remain invariant leads naturally to the same value for the first temporal moment.

Now only two independent parameters remain to be identified, $\left(\varphi_{\mathrm{m}}\right.$ and $\left.D_{\mathrm{m}}^{\prime}\right)$. To that end, we impose the exact identification of the second and third order moments; that is, Eqs. (8b), (8c) and (16b), (16c).
This process results in:

$D_{\mathrm{m}}^{\prime}=\frac{F}{6 E} \frac{\langle U\rangle}{a_{11}}$,

$\varphi_{\mathrm{m}}=\frac{F}{3 E^{2}} \varphi_{\mathrm{f}}$

These expressions, as well as the resulting ones for $\varphi_{\mathrm{f}}$ and $U^{\prime}$ for the different matrix shape models, are presented in Table 3. Finally, for the fourth order moment is obtained by substituting the expressions for $\varphi_{\mathrm{m}}, \varphi_{\mathrm{f}}$ and $D_{\mathrm{m}}^{\prime}$ into Eq. (16d). This leads to:

$M_{4, \tau}(x)=\frac{4 G}{E^{2}} \frac{a_{11}^{2}}{\langle U\rangle^{4}} x^{2}+\frac{72 E H}{F^{2}} \frac{a_{11}^{3}}{\langle U\rangle^{4}} x$.
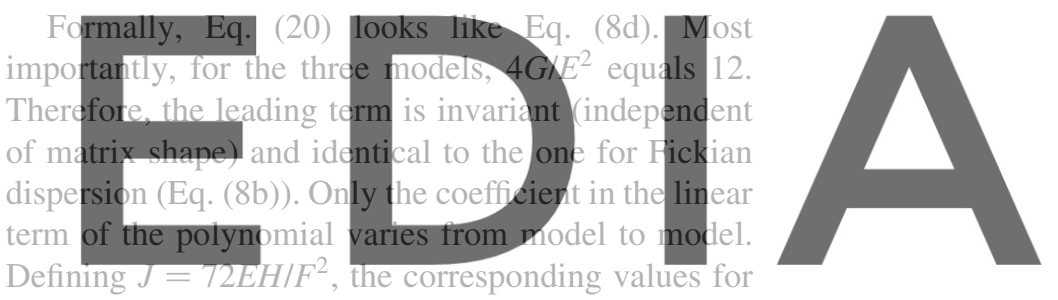

the three models can be seen in Table 3 .

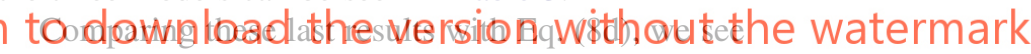

that the set of parameters that leads to identical first three temporal moments also leads to an exact identification of the leading term in the fourth order, while the linear term in Eq. (8d) is not well reproduced (giving values in the range $(97,101)$, depending on the model considered when the coefficient for the ADE equation was 120). It should also be noticed that the $J$ values are quite similar,

Table 3

Values for the different parameters that would lead to a total identification of the three first moments of the BTC plus the leading term of the fourth order

\begin{tabular}{|c|c|c|c|c|c|}
\hline Matrix shape & $\varphi_{\mathrm{m}}$ & $D_{\mathrm{m}}^{\prime}$ & $\varphi_{\mathrm{f}}$ & $U^{\prime}$ & $J$ \\
\hline Slabs & $\varphi_{\mathrm{m}}=\frac{3}{5} \varphi_{\mathrm{f}}$ & $D_{\mathrm{m}}^{\prime}=\frac{1}{5} \frac{\langle U\rangle}{a_{11}}$ & $\varphi_{\mathrm{f}}=\frac{2}{5} \varphi$ & $U^{\prime}=\frac{5}{2}\langle U\rangle$ & $680 / 7$ \\
\hline Cylinders & $\varphi_{\mathrm{m}}=\frac{2}{3} \varphi_{\mathrm{f}}$ & $D_{\mathrm{m}}^{\prime}=\frac{1}{12} \frac{\langle U\rangle}{a_{11}}$ & $\varphi_{\mathrm{f}}=\frac{1}{3} \varphi$ & $U^{\prime}=3\langle U\rangle$ & 99 \\
\hline Spheres & $\varphi_{\mathrm{m}}=\frac{5}{7} \varphi_{\mathrm{f}}$ & $D_{\mathrm{m}}^{\prime}=\frac{1}{21} \frac{\langle U\rangle}{a_{11}}$ & $\varphi_{\mathrm{f}}=\frac{2}{7} \varphi$ & $U^{\prime}=\frac{7}{2}\langle U\rangle$ & $504 / 5$ \\
\hline
\end{tabular}


so that any particular BTC that could be interpreted using any of the models, would allow using any other model for matrix diffusion with a different set of parameters. This is valid only for large travel distances, where the linear term is less relevant. Close to the inlet boundary the linear term is dominant, leading to the well known shape difference between the BTCs when a matrix diffusion or a macrodispersion term are considered.

4.2. Analysis and physical interpretation

of the parameters

We discuss now some potential physical interpretation of the parameters obtained in the identification

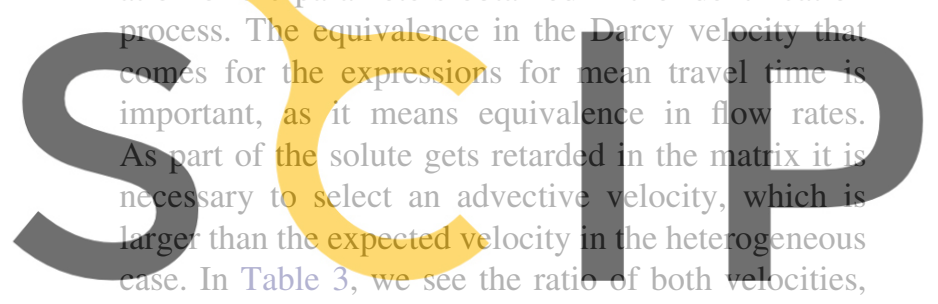

which ranges between $[5 / 2,7 / 2]$ depending of the

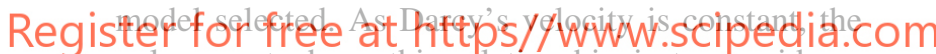

only way to have this relationship is to consider a mobile porosity, which is a small fraction of the total one. The values range from $2 / 5$ to $2 / 7$. Therefore, the immobile porosity is slightly larger than the mobile one for all models in this type of identification.

On the other hand $D_{\mathrm{m}}^{\prime}$ is a diffusion type parameter. It indicates the facility of the solute to move from the mobile to the immobile zone and vice versa. From Eq. (19a), it follows that $D_{\mathrm{m}}^{\prime}$ becomes inversely proportional to $a_{11}$, and directly proportional to $\langle U\rangle$. Regarding the latter, the reason is that large $\langle U\rangle$ will not allow diffusion to act long enough; then, only a small quantity of solute will go into the immobile zone, and the variance would decrease. The proportionality between $D_{\mathrm{m}}^{\prime}$ and $\langle U\rangle$ is of outmost importance. The main difference between dispersion and diffusion is precisely their dependence on velocity. Dispersion is caused by heterogeneous velocity and should be nearly invariant to changes in gradient. That is, if the regional gradient is multiplied by a factor of two, then all velocities should also be multiplied by that factor. The plume then will move twice as fast, but the dispersing paths will remain identical. As a result, the same plume shape will be reached, only twice as fast. Because of this, matrix diffusion fittings of BTCs have sometimes been regarded as fitting tricks, leading to models without predicting capabilities. What our findings suggests is that prediction capabilities will be maintained provided that matrix diffusion is taken as proportional to water flux, at least the portion of matrix diffusion aimed at representing dispersion.

Examination of the second moment of the BTCs yields the explanation for the inverse relationship between $D_{\mathrm{m}}^{\prime}$ and $a_{11}$. A large value of $a_{11}$ leads to a larger variance of travel time. The effect is the opposite for $D_{\mathrm{m}}^{\prime}$, as a large value tends to homogenize the concentrations at the mobile phase and the matrix, leading to a behavior closer to instantaneous equili-

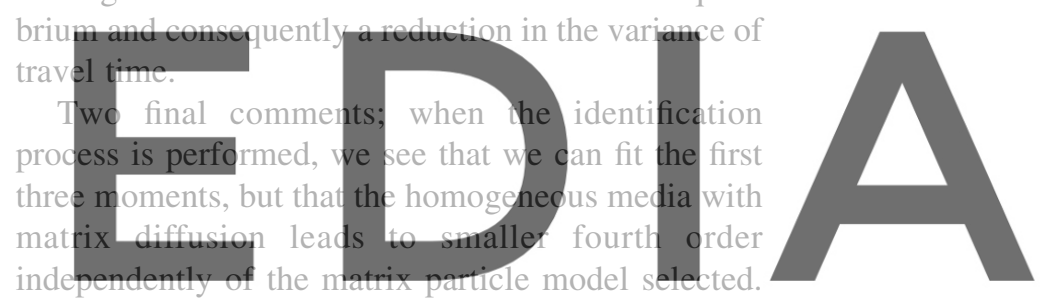

In any case, the difference decreases with increasing

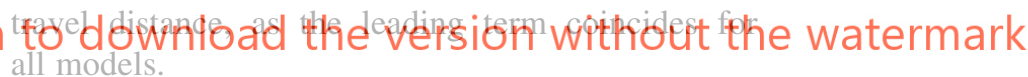

Last, it could be possible to extend the methodology to higher order moments. The probiem then would be the error associated to the evaluation of these terms. In general, the BTC has a considerable tailing. High order moments are then extremely uncertain.

\section{Conclusions}

The main conclusion from our work is that macrodispersion (or any kind of Fickian dispersion, for that matter) can be represented by means of a term that expresses exchange of solutes between mobile and immobile zones (MD). Using this term instead of the Fickian dispersion term of the conventional ADE allows reproducing the first three moments of the BTC and nearly so the fourth one.

Therefore, in principle, it would be possible to use indistinctly $\mathrm{MD}$ or $\mathrm{ADE}$ in order to represent macrodispersion in a given heterogeneous aquifer. One may even argue that the matrix-diffusion representation is more convenient than the Fickian 
representation of dispersion. Actually, it displays the asymmetry expected in spatial distribution of concentrations, which a Fickian representation does not. Also, it allows restricting Fickian dispersion to the local one, thus avoiding the negative side effects of large dispersivities (such as upstream dispersion). However, the proposed representation of dispersion fails to match the time evolution of the second-order moment of travel time. Therefore, while it might be convenient as an alternative for the macrodispersive ADE, it cannot avoid the fact that the fitted parameters would also be time dependent, thus restricting their applicability to real cases, same as a single value of dispersion is not valid to fit solute transport at different scales.

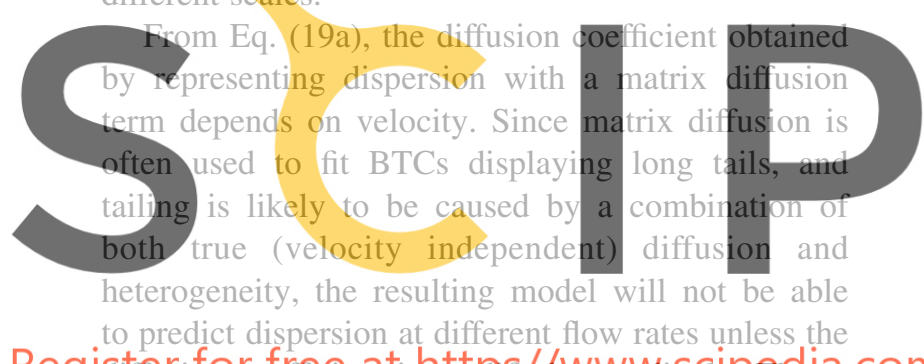

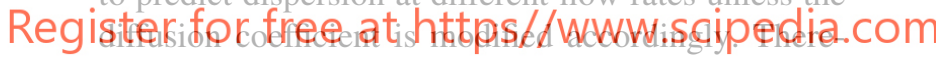

fore, one would need to perform tests with different

flow rates so as to discriminate the dependence on velocity of the fitting parameter or, in other words, the contribution of true diffusion and heterogeneity to transport.

Our results can also be seen from another point of view. When interpreting BTCs from tracer tests, hydrogeologists face the problem of having to infer the processes that the solute has undergone. The possibility of fitting similar temporal moments with different conceptual models shows that the simple study of the curve would not allow the identification of processes. While, we have studied only two conceptual models (MD and ADE), we must keep in mind that a solute that undergoes mass-transfer ratelimited processes that are controlled by reactions kinetics would have a BTC practically indistinguishable from that of a solute undergoing first order matrix diffusion (Selroos and Cvetkovic, 1992; Haggerty and Gorelick, 1995). Therefore, it would be possible to obtain a third set of parameters that could also fit a particular BTC with the same quality of fitting.

\section{Acknowledgements}

This work was partially funded by ENRESA (Spanish nuclear waste disposal company). The first author acknowledges support by the Department d'Universitats, Recerca i Societat de la Informació of the Catalan Government.

\section{References}

Aris, R., 1958. On the dispersion of linear kinematic waves. Proc. R. Soc. Lond. A 245, 268-277.

Barker, J.A., 1982. Laplace transform solutions for solute transport in fissured aquifers. Adv. Water Resour. 5, 98-104.
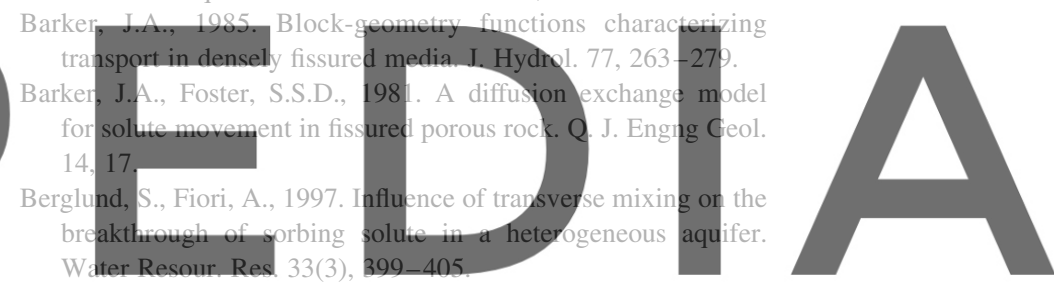

Berkowitz, B., Scher, H., 1995. On characterization of anomalous dispersion in porous and fractured media. Water. Resour. Res.

Butera, I., Tanda, M.G., 1999. Solute transport analysis through heterogeneous media in nonuniform in the average flow by a stochastic approach. Transp. Porous Miedia 36, 255-291.

Carrera, J., 1993. An overview of uncertainties in modelling groundwater solute transport. J. Contam. Hydrol. 13, 23-48.

Carrera, J., Samper, J., Galarza, G., Medina, A., 1990. An approach to process identification: Application to solute transport through clays, ModelCARE90: Calibration and Reliability in Groundwater Modelling, IAHS Publ. 195, pp. 231-240.

Carrera, J., Sánchez-Vila, X., Benet, I., Medina, A., Galarza, G., Guimerà, J., 1998. On matrix diffusion: formulations, solution methods and qualitative effects. Hydrogeol. J. 6, 178-190.

Cunningham, J.A., Werth, C.J., Reinhard, M., Roberts, P.V., 1997. Effects of grain-scale mass transfer on the transport of volatile organics through sediments. 1. Model development. Water Resour. Res. 33(12), 2713-2726.

Cushman, J.H., Ginn, T.R., 1993. Nonlocal dispersion in porous media with continuously evolving scales of heterogeneity. Transp. Porous Media 13, 123-138.

Cvetkovic, V., Shapiro, A.M., Dagan, G., 1992. A solute flux approach to transport in heterogeneous formations, 1. Model development. Water Resour. Res. 28(5), 1377-1388.

Cvetkovic, V., Cheng, H., Wen, X.-H., 1996. Analysis of nonlinear effects on tracer migration in heterogeneous aquifers using Lagrangian travel time statistics. Water Resour. Res. 32(6), $1671-1680$.

Dagan, G., 1984. Solute transport in heterogeneous porous formations. J. Fluid Mech. 14, 151-177. 
Dagan, G., 1987. Theory of solute transport by groundwater. Ann. Rev. Fluid Mech. 19, 183-215.

Dagan, G., 1988. Time-dependent macrodispersion for solute transport in anisotropic heterogeneous aquifers. Water Resour. Res. 24, 1491-1500.

Dagan, G., 1989. Flow and Transport in Porous Formations, Springer, New York, p. 465.

Dagan, G., Neuman, S.P., 1991. Nonasymptotic behavior of a common Eulerian approximation for transport in random velocity fields. Water Resour. Res. 27(12), 3249-3256.

Dentz, M., Kinzelbach, H., Attinger, S., Kinzelbach, W., 2000. Temporal behavior of a solute cloud in a heterogeneous porous medium. 1. Point-like injection. Water Resour. Res. 36(12), 3591-3604.

Gelhar, L.W., 1993. Stochastic Subsurface Hydrology, Prentice Hall, New Jersey, p. 390.

Gelhar, L.W., Axness, C.L., 1983. Three-dimensional stochastic analysis of macrodispersion in aquifers. Water Resour. Res. 19(1), 161-180.

Goltz, M.N., Roberts, P.V., 1987. Using the method of moments to analyze three-dimensional diffusion-limited solute transport from temporal and spatial perspectives. Water Resour. Res. 23(8), 1575-1585.

Gradshteyn, I.S., Ryzhik, I.M., 1980. Table of Integrals, Series, and Products, Academic Press, San Diego, p. 1160.

Grisak, G.E., Pickens, J.F., 1981. An analytical solution for solute transport through fractured media with matrix diffusion. J. Hydrol. 52, 47-57.

Guadagnini, A., Neuman, S.P., 2001. Recursive conditional moment equations for advective transport in randomly heterogeneous velocity fields. Transp. Porous Media 42, 37-67.

Guswa, A.J., Freyberg, D.L., 2000. Slow advection and diffusion through low permeability inclusions. J. Contam. Hydrol. 46, $205-232$.

Guswa, A.J., Freyberg, D.L., 2002. On using the equivalent conductivity to characterize solute spreading in environments with low permeability lenses. Water Resour. Res. 38(8), doi: 10.1029/2001WR000528.

Haggerty, R., Gorelick, S.M., 1995. Multiple-rate mass transfer for modeling diffusion and surface reactions in media with pore-scale heterogeneity. Water Resour. Res. 31(10), 2383-2400.

Haggerty, R., Gorelick, S.M., 1998. Modeling mass transfer processes in soil columns with pore-scale heterogeneity. Soil Sci. Soc. Am. J. 62(1), 62-74.

Harvey, C.F., Gorelick, S.M., 1995. Temporal moment-generating equations: modeling transport and mass transfer in heterogeneous aquifers. Water Resour. Res. 31(8), 1895-1912.

Jaekel, U., Vereecken, H., 1997. Renormalization group analysis of macrodispersion in a directed random flow. Water Resour. Res. 33, 2287-2299.

Kavvas, M.L., Karakas, A., 1996. On the stochastic theory of solute transport by unsteady and steady groundwater flow in heterogeneous aquifers. J. Hydrol. 179, 321-351.

Kennedy, C., Lennox, W.C., 1995. A control volume model of solute transport in a single fracture. Water Resour. Res. 31(2), $313-322$.
Kennedy, C., Lennox, W.C., 2001. A stochastic interpretation of the tailing effect in solute transport. Stochast. Environ. Res. Risk Assess. 15, 325-340.

Lunati, I., Attinger, S., Kinzelbach, W., 2002. Macrodispersivity for transport in arbitrary nonuniform flow fields: asymptotic and preasymptotic results. Water Resour. Res. 30(10), 1187, doi:10.1029/201WR001203.

Maloszewski, P., Zuber, A., 1985. On the theory of tracer experiments rocks with a porous matrix. J. Hydrol. 79, 333-358.

Maloszewski, P., Zuber, A., 1993. Tracer experiments in fractured rocks: Matrix diffusion and validity of models. Water Resour. Res. 29(8), 2723-2735.

Matheron, G., 1971. The theory of regionalized variables and its applications, Les Cahiers du CMM, Fasc. No. 5 ENSMP, Paris, p. 211.

McKenna, S.A., Meigs, L.C., Haggerty, R., 2001. Tracer tests in a fractured dolomite. 3. Double-porosity, multiple-rate mass transfer processes in convergent flow tracer tests. Water Resour. Res. 37(5), 1143-1154.

McLaughlin, D., Ruan, F., 2001. Macrodispersivity and largescale hydrogeologic variability. Transp. Porous Media 42, $133-154$.

Moench, A., 1984. Double porosity models for a fissured groundwater reservoir with fracture skin. Water Resour. Res. 20(7), 831-846.

Neretnieks, I., 1980. Diffusion in rock matrix: An important factor in radionuclide retardation? J. Geol. Res. 85(B8), 4379-4397.

Neuman, S.P., 1993. Eulerian-Lagrangian theory of transport in space-time nonstationary velocity fields: exact nonlocal formalism by conditional moments and weak approximation. Water Resour. Res. 29(3), 633-645.

Neuman, S.P., Zhang, Y.K., 1990. A quasi-linear theory of nonFickian subssurface dispersion. Theoretical analysis with application to isotropic media. Water Resour. Res. 26, 887-902.

Neuman, S.P., Winter, C.L., Newman, C.M., 1987. Stochastic theory of field-scale Fickian dispersion in anisotropic porous media. Water Resour. Res. 23(3), 453-466.

Novakowski, K.S., Lapceviec, P.A., 1994. Field measurement of radial solute transport in fractured rock. Water Resour. Res. 30(1), 37-44.

Rasmuson, A., 1984. The influence of particle shape on the dynamics of fixed beds. Chem. Engng Sci..

Rasmuson, A., Neretnieks, I., 1980. Exact solution of a model for diffusion in particles and longitudinal dispersion in packed beds. AIChE J. 26, 686.

Rasmuson, A., Neretnieks, I., 1981. Migration of radionuclides in fissured rocks-the influence of micropore diffusion and longitudinal dispersion. J. Geol. Res. 86, 3749-3758.

Rubin, Y., 2003. Applied Stochastic Hydrogeology, Oxford University Press, Amsterdam.

Schwarze, H., Jaekel, U., Vereecken, H., 2001. Estimation of macrodispersion by different approximation methods for flow and transport in randomly heterogeneous media. Transp. Porous Media 43, 265-287.

Selroos, J.-O., Cvetkovic, V., 1992. Modeling solute advection coupled with sorption kinetics in heterogeneous formations. Water Resour. Res. 28(5), 1271-1278. 
Shapiro, A.M., 2001. Effective matrix diffusion in kilometer-scale transport in fractured crystalline rock. Water Resour. Res. 37(3), 507-522.

Sposito, G., White, R.E., Darrah, P.R., Jury, W.A., 1986. A transfer function model of solute transport through soil. 3 . The convectiondispersion equation. Water Resour. Res. 22(2), 255-262.

Sudicky, E.A., Frind, E.O., 1992. Contaminant transport in fractured porous media: analytical solutions for a system parallel fractures. Water Resour. Res. 18(6), 1634-1642.

UPC-UPM, 1991. Síntesis de estudios hidrogeológicos para la modelación del flujo del agua subterránea en el entorno de las instalaciones de El Cabril (in Spanish), Report for Enresa, UPC, Barcelona.

Wood, W.W., Kraemer, T.F., Hearn, P.P., 1990. Intergranular diffusion: an important mechanism influencing solute transport in classic aquifers? Science 247, 1569-1572.

Wörman, A., Xu, S., Dverstorp, B., 2003. Kinematic analysis of solute mass flows in rock fractures with spatially random parameters. J. Contam. Hydrol. 60, 163-191.
Zhan, H., Wheatcraft, S.W., 1996. Macrodispersivity tensor for nonreactive solute transport in isotropic and anisotropic fractal porous media: analytical solutions. Water Resour. Res. 32(12), 3461-3474.

Zhang, D., 2002. Stochastic Methods for Flow in Porous Media: Coping with Uncertainties, Academic Press, New York.

Zimmerman, M.D., Bennett, P.C., Sharp, J.M. Jr., Choi, W.-J., 2002. Experimentaql determination of sorption in fractured flow systems. J. Contam. Hydrol. 58, 51-77.

Zinn, B., Harvey, C.F., 2003. When good statistical models of aquifer heterogeneity go bad: a comparison of flow, dispersion, and mass transfer in connected and multivariate Gaussian hydraulic conductivity fields. Water Resour. Res. 39(3), doi: 10.1029/2001WR001146

Zuber, A., Michalczyk, Z., Maloszewski, P., 2001. Great tritium ages explain the occurrence of good-quality groundwater in a phreatic aquifer of an urban area, Lublin, Poland. Hydrogeol. J. $9,451-460$. 